\title{
Applying the dynamic transmission electron microscope to study fast processes in materials
}

Geoffrey H. Campbell, Thomas B. Lagrange, Wayne E. King, Nigel D. Browning, Michael R. Armstrong, Judy S. Kim, Bryan W. Reed, Alan M. Frank, Brent C. Stuart, William J. DeHope, Benjamin J. Pyke, Richard M. Shuttlesworth, Frederic V. Hartemann, and David J. Gibson

University of California, Lawrence Livermore National Laboratory, Livermore, CA 94550

Ultrafast optical methods have been effectively used in chemistry and biology to understand the complex transient events that carry a reactant to a product state. More recently, x-rays have grown in importance as an ultrafast probe and their application has been extended to materials. Applications include the study of the time evolution of phase transitions in condensed matter, gas phase chemical reactions, and the study of excited (partially unfolded) proteins, i.e., the applications cross the disciplines of chemistry, biology, and materials science. The observation of intermediate states is of particular importance to gain insight into reaction or transformation pathways. Much less attention has been given to approaches based on laser induced and electron interrogation methods [1-3], despite the fact that electron sources are brighter and their interactions with matter stronger. Use of electrons as probes has demonstrated effectiveness [4] and great potential to study complex transient events not only because of the possible high temporal resolution, but also the potential for high spatial resolution imaging using the dynamic transmission electron microscopy (DTEM).

In this presentation, we discuss progress toward the development of a DTEM at Lawrence Livermore National Laboratory. We will present the anatomy of the DTEM instrument (See Figure 1). This includes discussions of the laser activated electron gun (also known as a photoelectron gun), the specimen drive provided by a second laser and its timing with the cathode laser, and the signal recording on a detector system. Practical aspects of operation will also be discussed, including determination of time-zero, measurement of pulse-length, and specimen response to the drive laser. We seek to study complex transient phenomena in situ by applying this high time resolution technique to fast, dynamic events in materials science. We have used specimen drive pulses of a few $\mu \mathrm{J}$ to heat specimens of hcp $\mathrm{Ti}, \alpha$-phase into the bcc, $\beta$-phase field and have observed their structural transformation with pulsed electron diffraction [5]. We study the kinetics of the transformation by monitoring the intensity of the diffracted signals coming from both phases as a function of time delay from the laser drive treatment. We also plan on measuring the kinetics of the transformation as a function of the temperature above the transformation temperature. With images, we will gain information on nucleation and on interphase boundary motion. We also anticipate applying this technique to study the reaction pathways and kinetics of reactive multilayer films and to the complex crystal structure transitions that accompany conformational changes in molecular systems.

UEM has the potential to make a significant impact in future science and technology. Understanding of reaction pathways of complex transient phenomena in materials science, biology, and chemistry will provide fundamental knowledge that will drive new discovery-class science. [6] 
References

[1] O. Bostanjoglo, Advances in imaging and electron physics 121 (2002) 1.

[2] O. Bostanjoglo, et al., Ultramicroacopy 81 (2000) 141.

[3] W.E. King, et al., J. Appl. Phys. 97 (2005) 111101.

[4] H. Dömer and O. Bostanjoglo, Adv. Eng. Mater. 4 (2002) 623.

[5] G.H. Campbell, et al., in Proceedings of the Solid-Solid Phase Transformations in Inorganic Materials 2005, Vol. 2, ed. J.M. Howe, et al., TMS, Warrendale, PA, (2005) 443.

[6] This work performed under the auspices of the Office of Basic Energy Sciences, U.S. Department of Energy by the University of California, Lawrence Livermore National Laboratory under contract No. W-7405-Eng-48. UCRL-ABS-218943.

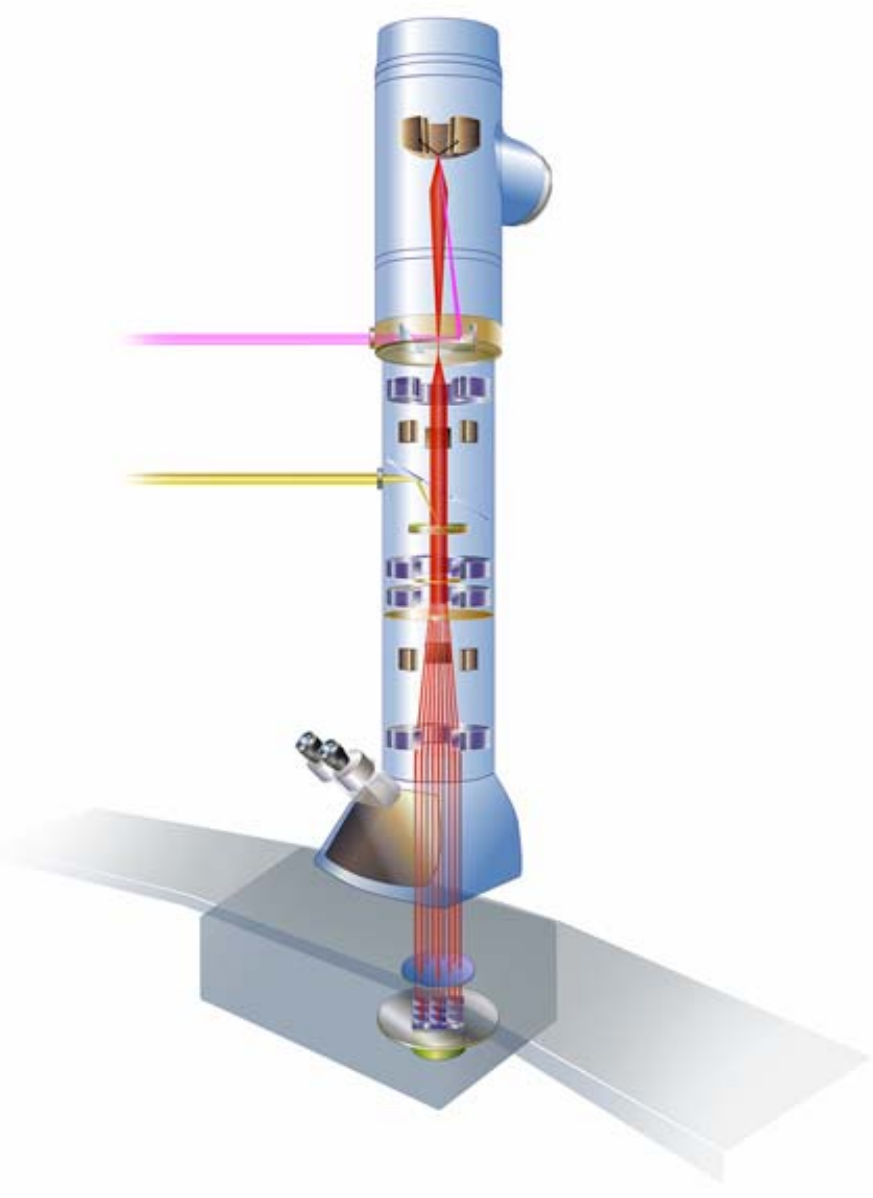

FIG. 1. Artists rendition of the LLNL DTEM. Two external lasers are introduced into the microscope column. The upper laser has a wavelength of $213 \mathrm{~nm}$ and is directed to the cathode to stimulate photoemission of the electrons. The second, lower laser has wavelength of $532 \mathrm{~nm}$ and drives a response in the specimen. 